\title{
A combination of two ELISA tests for nasopharyngeal carcinoma screening in endemic areas based on a case-control study
}

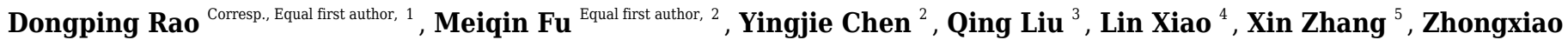
$\mathrm{Li}^{2}$, Haitao $\mathrm{Li}^{2}$, Yongyi He ${ }^{1}$, Yongxing Chen ${ }^{6}$, Jieying Chen ${ }^{2}$, Jin $\mathrm{Hu}^{2}$, Yanming Huang ${ }^{\text {Corresp. } 7}$

${ }^{1}$ Department of Medical Records, Jiangmen Central Hospital,Affiliated Jiangmen Hospital of Sun Yat-sen University, Jiangmen, Guangdong, China

2 Clinical Laboratory, Jiangmen Central Hospital, Affiliated Jiangmen Hospital of Sun Yat-sen University, Jiangmen, Guangdong, China

3 Department of Preventive Medicine, Sun Yat-sen University Cancer Center, Guangzhou, Guangdong, China

4 Department of Radiotherapy, Jiangmen Central Hospital,Affiliated Jiangmen Hospital of Sun Yat-sen University, Jiangmen, Guangdong, China

${ }^{5}$ Clinical Experimental Center,Jiangmen key Laboratory of Clinical Biobanks and Translational Research, Jiangmen Central Hospital,Affiliated Jiangmen Hospital of Sun Yat-sen University, Jiangmen, Guangdong, China

${ }^{6}$ Department of Ear-Nose-Throat, Jiangmen Central Hospital,Affiliated Jiangmen Hospital of Sun Yat-sen University, Jiangmen, Guangdong, China

7 Department of Respiratory Medicine,Clinical Experimental Center,jiangmen key Laboratory of Clinical Biobanks and Translational Research, Jiangmen Central Hospital,Affiliated Jiangmen Hospital of Sun Yat-sen University, Jiangmen, Guangdong, China

Corresponding Authors: Dongping Rao, Yanming Huang

Email address: a113202660@126.com, huangyanmingjxy@163.com

For populations with a high risk of nasopharyngeal carcinoma (NPC) in Guangdong province in southern China, mass screening is the first choice to prevent death from NPC. To improve the performance of NPC screening, we used a combination based on the IgA antibody against the Epstein-Barr virus (EBV) capsid antigen (VCA-IgA) and the IgA antibody against Epstein-Barr virus nuclear antigen 1 (EBNA1-IgA) to NPC screening by enzyme-linked immunosorbent assay (ELISA). A multiplication model was applied to measure the level of the combination. We evaluated the NPC screening effect of the markers. A case-control study was performed to assess the NPC screening effect of the markers. A total of 10,894 serum specimens were collected, including 554 samples from NPC patients and 10,340 samples from healthy controls. In the training stage, 640 subjects were randomly selected, including 320 NPC cases and 320 healthy controls. In the verification stage, 10,254 subjects were used to verify the NPC screening effect of the combination. Receiver operating characteristic (ROC) analysis was performed. In the verification stage, the combination achieved an sensitivity of $91.45 \%$, a specificity of $93.45 \%$, and an area under the ROC curve (AUC) of 0.978 (95\% Cl: $0.968,0.987)$. Compared with VCA-IgA and EBNA1-IgA individually, the combination had an improved screening performance. A probability (PROB) calculated by logistic regression model based on VCA-IgA and EBNA1-IgA was applied to NPC screening by ELISA in China. The AUC of the combination was a little bit larger than the PROB. There was a slight increase $(3.13 \%)$ in 
the sensitivity of the combination compared to the sensitivity of the PROB, while the specificity was lower for the combination (92.50\%) than for the PROB (95.94\%). We successfully applied a combination of two ELISA tests based on VCA-IgA and EBNA1-IgA for NPC screening by using a multiplication model. The results suggested that the combination was effective and can be an option for NPC screening. 
2 A combination of two ELISA tests for nasopharyngeal carcinoma screening in

3

4

5

6

7

\section{endemic areas based on a case-control study}

Dongping $\mathrm{Rao}^{1 \dagger^{*}}$, Meiqin $\mathrm{Fu}^{2 \dagger}$, Yingjie Chen ${ }^{2}$, Qing $\mathrm{Liu}^{3}$, Lin $\mathrm{Xiao}^{4}$, Xin Zhang 5 ,

Zhongxiao $\mathrm{Li}^{2}$, Haitao $\mathrm{Li}^{2}$, Yongyi $\mathrm{He}^{1}$, Yongxing $\mathrm{Chen}^{6}$, Jieying $\mathrm{Chen}^{2}$, Jin $\mathrm{Hu}^{2}$,

Yanming Huang $^{7 *}$

${ }^{1}$ Department of Medical Records, Jiangmen Central Hospital, Affiliated Jiangmen Hospital of Sun Yat-sen University, Jiangmen, Guangdong, China

${ }^{2}$ Clinical Laboratory, Jiangmen Central Hospital, Affiliated Jiangmen Hospital of Sun Yat-sen University, Jiangmen, Guangdong, China

${ }^{3}$ Department of Preventive Medicine, Sun Yat-sen University Cancer Center, Guangzhou, Guangdong, China

${ }^{4}$ Department of Radiotherapy, Jiangmen Central Hospital, Affiliated Jiangmen Hospital of Sun Yat-sen University, Jiangmen, Guangdong, China

${ }^{5}$ Clinical Experimental Center, Jiangmen key Laboratory of Clinical Biobanks and Translational Research, Jiangmen Central Hospital, Affiliated Jiangmen Hospital of Sun Yat-sen University, Jiangmen, Guangdong, China. ${ }^{6}$ Department of Ear-Nose-Throat, Jiangmen Central Hospital, Affiliated Jiangmen Hospital of Sun Yat-sen University, Jiangmen, Guangdong, China ${ }^{7}$ Department of Respiratory Medicine, Clinical Experimental Center, Jiangmen key Laboratory of Clinical Biobanks and Translational Research, Jiangmen Central Hospital, Affiliated Jiangmen Hospital of Sun Yat-sen University, Jiangmen, Guangdong, China

$\dagger$ These authors contributed equally to this article. 
"Corresponding authors:

31 Yanming Huang

32 No.23, Beijie Haibang Street, Pengjiang District, Jiangmen, Guangdong, 529030,

33 China

34 Email: huangyanming_jxy@163.com.

35 Dongping Rao

36 No.23, Beijie Haibang Street, Pengjiang District, Jiangmen, Guangdong, 529030,

37 China

38 Email: a113202660@126.com.

\section{Abstract}

For populations with a high risk of nasopharyngeal carcinoma (NPC) in Guangdong province in southern China, mass screening is the first choice to prevent death from NPC. To improve the performance of NPC screening, we used a combination based on the IgA antibody against the Epstein-Barr virus (EBV) capsid antigen (VCA-IgA) and the IgA antibody against Epstein-Barr virus nuclear antigen 1 (EBNA1-IgA) to NPC screening by enzyme-linked immunosorbent assay (ELISA). A multiplication model was applied to measure the level of the combination. We evaluated the NPC screening effect of the markers. A casecontrol study was performed to assess the NPC screening effect of the markers. A total of 10,894 serum specimens were collected, including 554 samples from NPC patients and 10,340 samples from healthy controls. In the training stage, 640 subjects were randomly selected, including 320 NPC cases and 320 healthy controls. In the verification stage, 10,254 subjects were used to verify the NPC screening effect of the combination. Receiver operating characteristic (ROC) analysis was performed. In the verification stage, the combination achieved an 
57 sensitivity of $91.45 \%$, a specificity of $93.45 \%$, and an area under the ROC curve (AUC) of 0.978 (95\% CI: 0.968, 0.987). Compared with VCA-IgA and EBNA1IgA individually, the combination had an improved screening performance. A probability (PROB) calculated by logistic regression model based on VCA-IgA and EBNA1-IgA was applied to NPC screening by ELISA in China. The AUC of the combination was a little bit larger than the PROB. There was a slight increase $(3.13 \%)$ in the sensitivity of the combination compared to the sensitivity of the PROB, while the specificity was lower for the combination $(92.50 \%)$ than for the PROB (95.94\%). We successfully applied a combination of two ELISA tests based on VCA-IgA and EBNA1-IgA for NPC screening by using a multiplication model. The results suggested that the combination was effective and can be an option for NPC screening.

\section{Introduction}

In southern China and southeast Asia, nasopharyngeal carcinoma (NPC) is a common malignant tumour, with an incidence rate of 10-40/100,000 per year $(\mathrm{Ng}$ et al., 2005; Torre et al., 2015; Wei \& Sham 2005; Yang et al., 2005; Yu \& Yuan 2002). Jiangmen city, an endemic area of NPC located along the Zhujiang River in the central southern area of Guangdong province, has a high-risk NPC population. The NPC incidence rate in the Jiangmen urban area is $14.99 / 10^{5}$ (Wei et al., 2017). The population-based cancer registry was established in Jiangmen to report the incidence and mortality of cancers. Population-based NPC screening was performed in the Jiangmen urban area by Jiangmen Central Hospital from June 2018 to March 2020.

The occurrence of NPC is strongly associated with Epstein-Barr virus (EBV) infection (Fachiroh et al., 2004; Gulley, 2001; Henle \& Henle, 1976; Sam, Abu- 
84 Samah \& Prasad,1994). Furthermore, host genetics, smoking, the consumption of

85 salted fish and occupational exposures are contributors to the pathogenesis of NPC

86 (Chang \& Adami, 2006; Chang et al., 2017; Chen et al., 2019; Yong et al., 2017).

87 The development mechanisms of NPC are unclear. Mass screening is the main

88 effective measure to detect NPC early in endemic areas.

89

90

EBV antibodies are widely used as markers in NPC screening (Chien et al., 2001; Ji et al., 2019; Ji et al., 2007; Ng et al., 2005; Tan et al., 2020; Zeng et al., 1982). A number of studies have shown that screening for NPC by using EBV antibodies is an effective measure to improve the survival rate of NPC patients (Choi et al., 2011; Ji et al., 2007; Jia et al., 2006; Ng et al., 2010). The combined serological test based on the IgA antibody against the EBV capsid antigen (VCAIgA) and the IgA antibody against EBV nuclear antigen 1 (EBNA1-IgA) by enzyme-linked immunosorbent assay (ELISA) was used for NPC screening in endemic areas in China (Gao et al., 2017; Liu et al., 2012; Yu et al., 2018). In previous studies, the probability (PROB) calculated by logistic regression based on VCA-IgA and EBNA1-IgA was applied to NPC screening in China (Gao et al., 2017; Liu et al., 2012; Yu et al., 2018).

Multiplication model was applied to make new maker to improve diagnostic effect (Attila et al., 2020). In this study, a combination of two ELISA tests based on VCA-IgA and EBNA1-IgA was applied to improve the effect of NPC screening by using a multiplication model and the NPC screening effect of the markers was evaluated.

\section{Materials \& Methods}




\section{Study population}

112 A case-control study was performed to compare the effect of the NPC screening of

113 markers, including 554 NPC cases and 10340 healthy controls. This study included

114 the training stage and the verification stage. The inclusion criteria for NPC cases

115 included being histologically confirmed by biopsy, aged between 30 and 69 years,

116 and residing in Jiangmen. A total of 554 serum specimens were continuously

117 collected from NPC patients at Jiangmen Central Hospital from June 2018 to

118 March 2020. Among the 554 cases, 7 (1.26\%) participated in the NPC screening

119 program. NPC stages were classified according to the 2008 staging system of

120 China (Lin ZX et al., 2009). The stages were divided into early-stage (stage I and

121 stage II) and advanced-stage (stage III and stage IV) disease. A total of 554 cases

122 comprised 73 early-stage cases and 481 advanced-stage cases. A total of 320 NPC

123 training samples were randomly selected from the 554 NPC cases, and the

124 remaining 234 of 554 NPC cases were used as validation samples.

125

126 A total of 10,340 healthy controls were obtained from an NPC screening

127 programme performed in a population aged 30-69 years in the Jiangmen City

128 urban area from June 2018 to March 2020. The healthy controls resided in

129 Jiangmen of Guangdong province. A total of 320 training samples were randomly

130 selected from the 10340 healthy controls and were frequency matched to the 320

131 training NPC cases by age (5-year age groups) and sex. The remaining 10020 of

13210340 healthy controls were used as the validation samples.

133

134 The information on age, sex, smoking history and family history of NPC for the cases and healthy controls were collected by inquiring medical records and using a questionnaire survey. 


\section{Serological test}

140 In total, 10894 serum samples were collected and underwent serological tests in 141 separate batches at Jiangmen Central Hospital. The samples were separated and 142 stored at $-40^{\circ} \mathrm{C}$. In this study, the NPC screening markers included VCA-IgA, 143 EBNA1/IgA and combination. The antibodies VCA-IgA (Euroimmun, Lubeck, 144 Germany) and EBNA1-IgA (Zhongshan Bio-tech, Zhongshan, China) were tested 145 by ELISA on a TECAN Freedom EVOlyzer 200/8 platform according to the 146 manufacturer's specifications. EBNA1s in Zhongshan Bio-tech kit were produced 147 with purified recombinant peptide specified by EBV BKRF1 (72 kD) (He et al. 148 2018). The EBV VCAs in Euroimmun kit were obtained from the pyrolysis

The written informed consent was obtained from healthy controls. The serum samples of NPC patients were collected after clinical use which were exempted from informed consent. This study was approved by the Clinical Research Ethics Committee of the Jiangmen Central Hospital (2019-28). 


\section{Statistical analysis}

166 Categorical variables are described as numbers and percentages. Continuous 167 variables are shown as the means and standard deviations (SDs). The levels of

168

169

170

171

172

173

174

175

176

177

178

179

180

181

182

183

184

185

186

187

188

189

190

191

VCA-IgA, EBNA1-IgA, PROB and combination were compared by t tests in different population. Receiver operating characteristic (ROC) curve analysis was performed. The cut-off value of each marker was defined with the largest Youden Index selected from each ROC curve. The effects of the screening markers were measured using the sensitivity, specificity and area under the ROC curve (AUC). The base information of different populations was described and compared by the $\chi^{2}$ test and Fisher's exact test. The difference in sensitivities of markers were compared by $\chi^{2}$ test, Fisher's exact test and McNemar test.

The differences in AUCs were compared using the $\mathrm{Z}$ test according to the DeLong method ( DeLong et al., 1988). The 95\% confidence intervals (CIs) of the sensitivities, specificities and AUCs were calculated. The statistical analyses were carried out using MedCalc Statistical Software version 15.2.2 (MedCalc Software bvba, Ostend, Belgium) and GraphPad Prism software version 8.0 (San Diego, CA, USA) and were two-sided, with significance set at $\mathrm{p}<0.05$.

\section{Results}

\section{Baseline information}

The characteristics of the 554 cases and 10340 healthy controls are shown in Table 1. In total, 554 NPC patients were enrolled in this study. Of them, 397 (71.66\%) were men, and the mean age was $50.86 \pm 9.48$ years. Among the 554 patients, 198 (35.74\%) had a smoking history, and $56(10.11 \%)$ had a family history of NPC. Of the 10340 healthy controls, 3959 (38.29\%) were men, and the mean age was 48.57 
$192 \pm 11.60$ years. Among the 10340 healthy controls, $1670(16.15 \%)$ had a smoking

193 history, and $198(1.91 \%)$ had a family history of NPC (Table 1).

194

195

196

197

198

199

200

201

202

203

204

205

206

207

208

209

210

211

212

213

214

215

The characteristics of the 320 cases and 320 healthy controls in the training stage are shown in Table 2. In this stage, the controls and NPC cases were matched by sex and age to prevent bias. Differences in age, smoking history and sex were not statistically significant, while differences in NPC family history were significant between the cases and controls. There were no statistically significant differences in sex, age, smoking history, or NPC family history between the earlystage and advanced-stage cases (Table 2).

The differences in age, sex, smoking history and NPC family history were significant between NPC cases and healthy controls (Table 1). There were no statistically significant differences in age, sex, smoking history and NPC family history between the early-stage and advanced-stage cases (Table 1).

9


220 Comparison of levels of markers in early-stage and advanced-stage NPC 221 patients

222 Of the 554 NPC patients, 73 (13.18\%) were early-stage. The levels of VCA-IgA 223 EBNA1-IgA, PROB and combination in early-stage and advanced-stage NPC 224 patients were showed in Figure 2. The differences in VCA-IgA, EBNA1-IgA, 225 PROB and combination were not significant between early-stage and advanced226 stage NPC patients by $t$ tests $(\mathrm{p}>0.05)$.

\section{Diagnostic value of the markers}

230

The diagnostic performance of the markers is shown in Table 3 by using training

231 samples. The combination achieved a sensitivity of $90.94 \%$ (95\% CI: $87.2 \%$, 93.8\%), a specificity of $92.50 \%$ (95\% CI: 89.0\%, 95.1\%) and an AUC of 0.978 (95\% CI: $0.969,0.986)$. The PROB achieved a sensitivity of $87.81 \%$ (95\% CI: $83.7 \%, 91.2 \%)$, a specificity of 95.94\% (95\% CI: 93.2\%, 97.8\%) and an AUC of 0.972 (95\% CI: $0.962,0.982)$. The VCA-IgA had a sensitivity of $84.06 \%$ (95\% CI: $79.6 \%, 87.9 \%)$, a specificity of $91.25 \%$ (95\% CI: 87.6\%, 94.1\%) and an AUC of 0.947 (95\% CI: 0.932, 0.963). The sensitivity, specificity and AUC of EBNA1-IgA were $87.81 \%$ (95\% CI:83.7\%, 91.2\%), 85.00\% (95\% CI: 80.6\%, 88.7\%), and 0.935 (95\% CI: 0.917, 0.953), respectively.

Compared to the AUCs of VCA-IgA $(p<0.001)$,EBNA1-IgA $(p<0.001)$, and PROB $(\mathrm{p}<0.01), \quad$ the combination yielded a higher AUC (Table 3 and Figure 3$)$ by using training samples. The differences in the sensitivities of the markers between early-stage and advanced-stage NPC patients were not significant by using verification samples $(p>0.05$, Table 4$)$. Compared with each marker alone by 
246 McNemar test, the combination had a higher sensitivity for early-stage NPC

247 patients (Table 4).

The differences in sensitivities of EBNA1-IgA, PROB and the combination between man and female NPC patients were not significant by using verification samples ( $\mathrm{p}>0.05)$, while the sensitivity of VCA-IgA in man NPC patients was higher than in female NPC patients ( $\mathrm{p}=0.047$, Table 5 ). The sensitivity differences of the markers in different age, smoking history and NPC family history were not statistically significant by by using verification samples $(p>0.05$, Table 6 , Table 7 , 255 Table 8).

\section{Verifying the effect of the combination on NPC screening}

258

259

260

261

262

263

264

265

266

267

268

269

270

271

272

A total of 10253 subjects were enrolled to verify the NPC screening effect, including 234 NPC cases and 10020 healthy controls sourced from the screening field. In this stage, the combination achieved an overall sensitivity of $91.45 \%$ (214/234), a sensitivity for early-stage NPC detection of 93.94\% (31/33), a specificity of $93.45 \%$ (9364/10020), and an AUC of 0.978 (95\% CI: $0.968,0.987)$.

\section{Discussion}

NPC is a main health problem that leads to a high health burden in southern China, especially in Guangdong province (Cao et al., 2011). EBV antibodies are widely applied in NPC screening. PROB calculated by logistic model were applied in NPC screening based on VCA-IgA and EBNA1-IgA (Gao et al., 2017; Liu et al., 2013; Yu et al., 2018). In this study, a combination calculated by multiplication model based on VCA-IgA and EBNA1-IgA was applied to NPC screening. The NPC screening effect of combination was evaluated and compared with the 
273 individual screening markers, PROB, VCA-IgA and EBNA1-IgA. Compared with

274 PROB, VCA-IgA and EBNA1-IgA, the combination had a higher AUC of 0.978

275

276

277

278

279

280

281

282

283

284

285

286

287

288

289

290

291

292

293

294

295

296

297

298

299

(95\% CI: $0.969,0.986)$. We found that the combination calculated by using a multiplication model can be applied to NPC screening.

In this study, a large number of healthy controls and 554 NPC patients were collected from the endemic areas, which is favourable for evaluating the performance of the markers for NPC screening. In the verification stage, 10254 subjects were enrolled to verify the NPC screening effect. The combination achieved a sensitivity of $91.45 \%$, a specificity of $93.45 \%$ and an AUC of 0.978 (95\% CI: $0.968,0.987)$. These results demonstrated that the combination calculated by using a multiplication model was effective for NPC screening.

In this study, the levels of markers (PROB, VCA-IgA, EBNA1-IgA and combination) in NPC patients were higher than in healthy controls. It was consistent with the results of previous reports (Liu et al., 2012). We found the difference in sensitivities of the combination in different age, sex, smoking history and NPC family history were not statistically significant. The VCA-IgA had a higher sensitivity for man NPC patients than female NPC patients by using verification samples. Since the P value (0.047) was very close to 0.05 , and the verification sample size was not very large. The difference in sensitivity of VCAIgA between man and female NPC patients may be due to the random fluctuation.

In the present study, the AUCS, sensitivities and specificities of VCA-IgA and EBNA1-IgA were lower than those of the combination, showing that the combination was more effective in diagnosis. The AUC of the combination was a little bit larger than the PROB. There was a slight increase (3.13\%) in the 
300 sensitivity of the combination compared to the sensitivity of the PROB. The

301 specificity was lower for the combination (92.50\%) than for the PROB (95.94\%).

302 In areas with high NPC incidence, the increased sensitivity means that more early-

303 stage NPC patients will be detected and treated early, while the decreased

304 specificity may lead to an increased false positive rate and increased costs of the

305 screening program.

The present study had some limitations. First, there was some bias in identifying the 10340 subjects as healthy controls because not all healthy controls underwent an examination by fibreoptic endoscopic examination. Second, since the study population was obtained from provinces with a high risk of NPC, the results may be limited for application in other populations. Third, the sample size of the earlystage NPC patients was not large enough in this study. There was some bias in estimating sensitivity for early-stage NPC patients.

\section{Conclusions}

We successfully developed a combination of two ELISA tests based on VCA-IgA and EBNA1-IgA to improve the effect of NPC screening by using a multiplication model. Compared with VCA-IgA and EBNA1-IgA individually, the combination had an improved diagnostic performance. The AUC and sensitivity of the combination were slightly higher than those of the PROB, while the specificity was lower for the combination than for the PROB. The results suggested that the combination was effective and can be an option for NPC screening.

\section{Acknowledgements}


327 We thank the staff from Jiangmen Jianghai District People's Hospital (Chunlai

328 Zhang and Yanshuang Lu), Waihai Public Health Service Center (Guozheng Zhou

329 and Wenguang Lin), Jiangnan Public Health Service Center (Cannong Liang and

330 Chunhua Xiong), Hetang Public Health Service Center (Wenwei Li), and all the

331 other staff involved in this programme for their hard work.

References

Attila Enyedi, Alexandra Csongrádi, István T Altorjay, Gergő L

Beke, Csongor Váradi, Enikő E Enyedi, Dorina R Kiss, Emese Bányai, Edit Kalina, János Kappelmayer, Attila Tóth, Zoltán Papp, István Takács, Miklós Fagyas. 2020. Combined application of angiotensin converting enzyme and chitotriosidase analysis improves the laboratory diagnosis of

Cao SM, Simons MJ, Qian CN. 2011. The prevalence and prevention of nasopharyngeal carcinoma in China. Chin J Cancer 30(2):114-119 DOI 10.5732/cjc.010.10377.

Chang ET, Adami HO. 2006. The enigmatic epidemiology of nasopharyngeal carcinoma. Cancer Epidemiol Biomarkers Prev 15(10):1765-1777 DOI 10.1158/1055-9965.EPI-06-0353.

Chang ET, Liu Z, Hildesheim A, Liu Q, Cai Y, Zhang Z, Chen G, Xie SH, Cao SM, Shao JY, Jia WH, Zheng Y, Liao J, Chen Y, Lin L, Ernberg I, Vaughan TL, Adami HO, Huang G, Zeng Y, Zeng YX, Ye W. 2017. Active and Passive Smoking and Risk of Nasopharyngeal Carcinoma: A PopulationBased Case-Control Study in Southern China. Am J Epidemiol 185(12):12721280 DOI 10.1093/aje/kwx018. 
353 Chen YP, Chan ATC, Le QT, Blanchard P, Sun Y, Ma J. 2019.

354 Nasopharyngeal carcinoma. Lancet 394(10192):64-80 DOI 10.1016/S0140-

355 6736(19)30956-0.

356

357

358

359

360

361

362

363

364

365

366

367

368

369

370

371

372

373

374

375

376

377

378

379

Chien YC, Chen JY, Liu MY, Yang HI, Hsu MM, Chen CJ, Yang CS. 2001. Serologic markers of Epstein-Barr virus infection and nasopharyngeal carcinoma in Taiwanese men. $N$ Engl J Med 345(26):1877-1882 DOI 10.1056/NEJMoa011610.

Choi CW, Lee MC, Ng WT, Law LY, Yau TK, Lee AW. 2011. An analysis of the efficacy of serial screening for familial nasopharyngeal carcinoma based on Markov chain models. Fam Cancer 10(1):133-139 DOI 10.1007/s10689-0109397-7.

DeLong ER, DeLong DM, Clarke-Pearson DL. 1988. Comparing the areas under two or more correlated receiver operating characteristic curves: a nonparametric approach. Biometrics 44(3):837-845.

Fachiroh J, Schouten T, Hariwiyanto B, Paramita DK, Harijadi A, Haryana SM, Ng MH, Middeldorp JM. 2004. Molecular diversity of Epstein-Barr virus $\mathrm{IgG}$ and $\mathrm{IgA}$ antibody responses in nasopharyngeal carcinoma: a comparison of Indonesian, Chinese, and European subjects. J Infect Dis 190(1):53-62 DOI $10.1086 / 421245$.

Gao R, Wang L, Liu Q, Zhang LF, Ye YF, Xie SH, Du JL, Chen SH, Guo J, Yang MJ, Lin CY1, Cao SM. 2017. Evaluation of seven recombinant VCAIgA ELISA kits for the diagnosis of nasopharyngeal carcinoma in China: a casecontrol trial. BMJ Open 7(6):e013211 DOI 10.1136/bmjopen-2016-013211.

Gulley ML. 2001. Molecular diagnosis of Epstein-Barr virus-related diseases. $J$ Mol Diagn 3(1):1-10 DOI 10.1016/S1525-1578(10)60642-3.

He YQ, Xue WQ, Xu FH, Xu YF, Zhang JB, Yu HL, Feng QS, Chen LZ, Cao SM, Liu Q, Mu J, Zeng YX, Jia WH. 2018. The Relationship Between 
380 Environmental Factors and the Profile of Epstein-Barr Virus Antibodies in the

381 Lytic and Latent Infection Periods in Healthy Populations from Endemic and

382 Non-Endemic Nasopharyngeal Carcinoma Areas in China. EBioMedicine

383 30:184-191 DOI 10.1016/j.ebiom.2018.02.019.

384 Henle G, Henle W. 1976. Epstein-Barr virus-specific IgA serum antibodies as an 385 outstanding feature of nasopharyngeal carcinoma. Int J Cancer 17(1):1-7 DOI 10.1002/ijc.2910170102.

Ji MF, Huang QH, Yu X, Liu Z, Li X, Zhang LF, Wang P, Xie SH, Rao HL,

Evaluation of plasma Epstein-Barr virus DNA load to distinguish nasopharyngeal carcinoma patients from healthy high-risk populations in Southern China. Cancer 120(9):1353-1360 DOI 10.1002/cncr.28564. SF, Wang PP, Quan W, Deng L, Li XH, Liu XD, Xie YL, Huang SJ, Ge

2019. Incidence and mortality of nasopharyngeal carcinoma: interim analysis of 
406

407

408

409

410

411

412

413

414

415

416

417

418

419

420

421

422

423

424

425

426

427

428

429

430

431

432

2002) in Sihui and Cangwu counties in southern China. BMC Cancer 6:178 DOI 10.1186/1471-2407-6-178.

Lin ZX, Yang ZN, Zhan YZ, Xie WJ, Li GW, Feng HT. 2009. Application study of the 2008 staging system of nasopharyngeal carcinoma. Ai Zheng 28(10):1029-1032 DOI 10.5732/cjc.009.10431.

Liu Y, Huang Q, Liu W, Liu Q, Jia W, Chang E, Chen F, Liu Z, Guo X, Mo H, Chen J, Rao D, Ye W, Cao S, Hong M. 2012. Establishment of VCA and EBNA1 IgA-based combination by enzyme-linked immunosorbent assay as preferred screening method for nasopharyngeal carcinoma: a two-stage design with a preliminary performance study and a mass screening in southern China. Int J Cancer 131(2):406-416 DOI 10.1002/ijc.26380.

Liu Z, Ji MF, Huang QH, Fang F, Liu Q, Jia WH, Guo X, Xie SH, Chen F, Liu Y, Mo HY, Liu WL, Yu YL, Cheng WM, Yang YY, Wu BH, Wei KR, Ling W, Lin X, Lin EH, Ye W, Hong MH, Zeng YX, Cao SM. 2013. Two

Epstein-Barr virus-related serologic antibody tests in nasopharyngeal carcinoma screening: results from the initial phase of a cluster randomized controlled trial in Southern China. Am J Epidemiol 177(3):242-250 DOI 10.1093/aje/kws404.

Ng WT, Choi CW, Lee MC, Law LY, Yau TK, Lee AW. 2010. Outcomes of nasopharyngeal carcinoma screening for high risk family members in Hong Kong. Fam Cancer 9(2):221-228 DOI 10.1007/s10689-009-9296-y.

Ng WT, Yau TK, Yung RW, Sze WM, Tsang AH, Law AL, Lee AW. 2005. Screening for family members of patients with nasopharyngeal carcinoma. Int $J$ Cancer 113(6):998-1001 DOI 10.1002/ijc.20672.

Sam CK, Abu-Samah AJ, Prasad U. 1994. IgA/VCA as a follow-up marker in the monitoring of nasopharyngeal carcinoma. Eur J Surg Oncol 20(5):561-564.

Tan LP, Tan GW, Sivanesan VM, Goh SL, Ng XJ, Lim CS, Kim WR, Mohidin T, Mohd Dali NS, Ong SH, Wong CY, Sawali H, Yap YY, Hassan 
433

434

435

436

437

438

439

440

441

442

443

444

445

446

447

448

449

450

451

452

453

454

455

456

457

458

459

\section{F, Pua KC, Koay CE, Ng CC, Khoo AS; Malaysian Nasopharyngeal}

Carcinoma Study Group. 2020. Systematic comparison of plasma EBV

DNA, anti-EBV antibodies and miRNA levels for early detection and prognosis of nasopharyngeal carcinoma. Int J Cancer 146(8):2336-2347 DOI 10.1002/ijc.32656.

Torre LA, Bray F, Siegel RL, Ferlay J, Lortet-Tieulent J, Jemal A. 2015.

Global cancer statistics, 2012. CA Cancer J Clin 65(2):87-108 DOI 10.3322/caac. 21262 .

Wei KR, Zheng RS, Zhang SW, Liang ZH, Li ZM, Chen WQ. 2017.

Nasopharyngeal carcinoma incidence and mortality in China, 2013. Chin J

Cancer 36(1):90 DOI 10.1186/s40880-017-0257-9.

Wei WI, Sham JST. 2005. Nasopharyngeal carcinoma. The Lancet

365(9476):2041-2054 DOI 10.1016/S0140-6736(05)66698-6.

Yang XR, Diehl S, Pfeiffer R, Chen CJ, Hsu WL, Dosemeci M, Cheng YJ, Sun B, Goldstein AM, Hildesheim A, Chinese and American Genetic Epidemiology of NPC Study Team. 2005. Evaluation of risk factors for nasopharyngeal carcinoma in high-risk nasopharyngeal carcinoma families in Taiwan. Cancer Epidemiol Biomarkers Prev 14(4):900-905 DOI 10.1158/10559965.EPI-04-0680.

Yong SK, Ha TC, Yeo MC, Gaborieau V, McKay JD, Wee J. 2017. Associations of lifestyle and diet with the risk of nasopharyngeal carcinoma in Singapore: a case-control study. Chin J Cancer 36(1):3 DOI 10.1186/s40880016-0174-3.

Yu MC, Yuan JM. 2002. Epidemiology of nasopharyngeal carcinoma. Semin Cancer Biol 12(6):421-429 DOI 10.1016/s1044579x02000858.

Yu X, Ji M, Cheng W, Wu B, Du Y, Cao S. 2018. Assessment of the Long-term Diagnostic Performance of a New Serological Screening Scheme in Large-scale 
460 Nasopharyngeal Carcinoma Screening. J Cancer 9(12):2093-2097 DOI

$461 \quad 10.7150 /$ jca.23755.

462 Zeng Y, Zhang LG, Li HY, Jan MG, Zhang Q, Wu YC, Wang YS, Su GR.

463 1982. Serological mass survey for early detection of nasopharyngeal carcinoma

464 in Wuzhou City, China. Int J Cancer 29(2):139-141 DOI

$465 \quad 10.1002 / \mathrm{ijc} .2910290204$.

466 


\section{Table $\mathbf{1}$ (on next page)}

Characteristics of the total population 
1 Table 1. Characteristics of the total population

\begin{tabular}{|c|c|c|c|c|c|c|}
\hline \multirow{4}{*}{ Categories } & \multicolumn{4}{|c|}{ NPC cases $(\mathrm{N}=554), \mathrm{n}(\%)$} & \multicolumn{2}{|c|}{ Controls $(\mathrm{N}=10340)$} \\
\hline & Early-stage & \multicolumn{2}{|l|}{ Advanced- stage } & \multirow{3}{*}{$\mathrm{P}^{*}$} & \multirow{3}{*}{$\mathrm{n}(\%)$} & \multirow{3}{*}{$\mathrm{P}^{\dagger}$} \\
\hline & NPC cases & NPC & Total & & & \\
\hline & $(n=73)$ & $(n=481)$ & & & & \\
\hline Sex & & & & 0.356 & & $<0.001$ \\
\hline Male & $49(67.12)$ & $348(72.35)$ & 397(71.66) & & $3959(38.29)$ & \\
\hline Female & $24(32.88)$ & $133(27.65)$ & $157(28.34)$ & & $6381(61.71)$ & \\
\hline Age (years) & & & & 0.546 & & $<0.001$ \\
\hline $30 \sim$ & $7(9.60)$ & $26(5.41)$ & $33(5.96)$ & & $1405(13.59)$ & \\
\hline $35 \sim$ & $4(5.48)$ & $35(7.28)$ & $39(7.04)$ & & $1439(13.92)$ & \\
\hline $40 \sim$ & $6(8.22)$ & $64(13.31)$ & $70(12.64)$ & & $1366(13.21)$ & \\
\hline $45 \sim$ & $15(20.55)$ & $94(19.54)$ & $109(19.68)$ & & $1512(14.62)$ & \\
\hline $50 \sim$ & $14(19.18)$ & $86(17.88)$ & $100(18.05)$ & & $1244(12.03)$ & \\
\hline $55 \sim$ & $8(3.70)$ & $73(15.18)$ & $81(14.62)$ & & $997(9.64)$ & \\
\hline $60 \sim$ & $11(15.07)$ & $71(14.76)$ & $82(14.80)$ & & $878(8.49)$ & \\
\hline $65 \sim 69$ & $8(10.96)$ & $32(6.65)$ & $40(7.22)$ & & $1499(14.50)$ & \\
\hline \multicolumn{2}{|c|}{ Smoking history } & & & 0.584 & & $<0.001$ \\
\hline Yes & $24(32.88)$ & $174(36.17)$ & $198(35.74)$ & & $1670(16.15)$ & \\
\hline No & $49(67.12)$ & $307(63.83)$ & $356(64.26)$ & & $8670(83.85)$ & \\
\hline \multicolumn{2}{|c|}{ NPC family history } & & & 0.566 & & $<0.001$ \\
\hline Yes & $6(8.22)$ & $50(10.4)$ & $56(10.11)$ & & $198(1.91)$ & \\
\hline No & $67(91.78)$ & $431(89.60)$ & 498(89.89) & & $10142(98.09)$ & \\
\hline
\end{tabular}

$2{ }^{*}$ Differences in sex, age, smoking history and NPC family history between early-stage and advanced-stage NPC

3 cases were compared by the $\chi^{2}$ test.

$4 \quad$ Differences in the baseline information distributions of the NPC cases and controls were compared by the $\chi^{2}$ test. 


\section{Table 2 (on next page)}

Characteristics of the training stage population 
1 Table 2. Characteristics of the training stage population

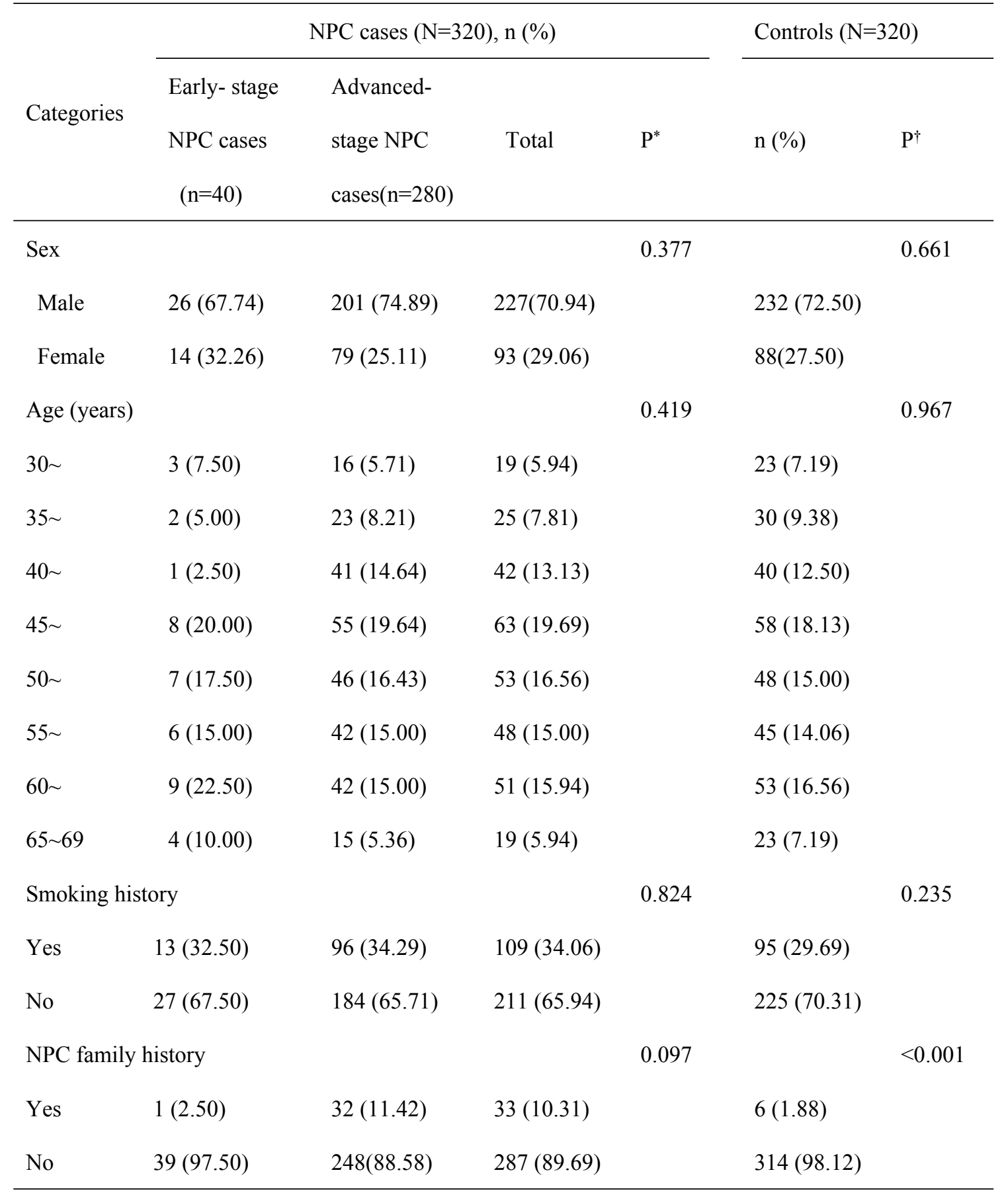

$2 \quad$ *Differences in sex and smoking history between early-stage and advanced-stage NPC cases were compared by the

$3 \chi^{2}$ test. Differences in age and NPC family history between early-stage and advanced-stage NPC cases were

4 compared by Fisher's exact test.

5 Differences in the baseline information distributions of the NPC cases and controls were compared by the $\chi^{2}$ test. 


\section{Table 3 (on next page)}

Diagnostic value of the markers 
1 Table 3. Diagnostic value of the markers

\begin{tabular}{cccccc}
\hline Marker & $\begin{array}{c}\text { Cut-off } \\
\text { value }\end{array}$ & $\begin{array}{c}\text { Sensitivity (\%) } \\
(95 \% \mathrm{CI})\end{array}$ & $\begin{array}{c}\text { Specificity }(\%) \\
(95 \% \mathrm{CI})\end{array}$ & $\begin{array}{c}\text { AUC }(95 \% \mathrm{CI}) \\
\mathrm{P}^{*}\end{array}$ \\
\hline VCA-IgA $\times$ & 0.429 & $90.94(87.2,93.8)$ & $92.50(89.0,95.1)$ & $0.978(0.969,0.986)$ & \\
EBNA1-IgA & & $87.81(83.7,91.2)$ & $95.94(93.2,97.8)$ & $0.972(0.962,0.982)$ & $<0.01$ \\
PROB & 0.949 & $84.06(79.6,87.9)$ & $91.25(87.6,94.1)$ & $0.947(0.932,0.963)$ & $<0.001$ \\
VCA-IgA & 1.194 & $87.81(83.7,91.2)$ & $85.00(80.6,88.7)$ & $0.935(0.917,0.953)$ & $<0.001$ \\
EBNA1-IgA & 0.397 & & & & \\
\hline
\end{tabular}

$2{ }^{*}$ The AUC comparisons of the markers were performed by the $\mathrm{Z}$ test.

3

4

5

6

7

8 


\section{Table 4(on next page)}

Sensitivity differences for early-stage and advanced-stage NPC by using different markers 
1 Table 4. Sensitivity differences for early-stage and advanced-stage NPC by using different markers

\begin{tabular}{ccccc}
\hline & & \multicolumn{2}{c}{ Sensitivity (\%) } \\
\cline { 3 - 4 } Marker & Cut-off value & Early-stage & Advanced- & $\mathrm{P}^{\dagger}$ \\
& & NPC cases & stage NPC cases & \\
\hline VCA-IgAx & 0.429 & 93.94 & 91.04 & 0.747 \\
EBNA1-IgA & & & & \\
PROB & 0.949 & $84.85^{f}$ & 84.08 & 1.000 \\
VCA-IgA & 1.194 & $75.76^{\#}$ & 82.59 & 0.339 \\
EBNA1-IgA & 0.397 & $84.85^{*}$ & 86.07 & 0.791 \\
\hline
\end{tabular}

2 The sensitivity differences between early-stage and advanced-stage NPC were compared by Fisher's exact test.

$3{ }^{f}$ Compared with combination, the PROB had a lower sensitivity for early-stage NPC patients by McNemar test

$4 \quad(\mathrm{p}<0.001)$. "Compared with combination, the VCA-IgA had a lower sensitivity for early-stage NPC patients by

5 McNemar test $(\mathrm{p}<0.001)$. ${ }^{*}$ compared with combination, the EBNA1-IgA had a lower sensitivity for early-stage NPC

6 patients by McNemar test $(\mathrm{p}<0.001)$.

7 


\section{Table 5 (on next page)}

Sensitivity differences for man and female NPC by using different markers 
1 Table 5. Sensitivity differences for man and female NPC by using different markers

\begin{tabular}{ccccc}
\hline \multirow{2}{*}{ Marker } & Cut-off value & \multicolumn{2}{c}{ Sensitivity (\%) for NPC cases } & \multirow{2}{*}{$\mathrm{P}^{\dagger}$} \\
\cline { 3 - 4 } & & Man & Female & \\
\hline VCA-IgAx & 0.429 & 91.76 & & \\
EBNA1-IgA & & & 90.63 & 0.781 \\
PROB & 0.949 & 85.29 & 81.25 & 0.450 \\
VCA-IgA & 1.194 & 84.71 & 73.44 & 0.047 \\
EBNA1-IgA & 0.397 & 85.88 & 85.93 & 0.991 \\
\hline
\end{tabular}

2 The sensitivity differences between man and female NPC were compared by $\chi^{2}$ test. 


\section{Table 6(on next page)}

Sensitivity differences for different ages of NPC patients by using different markers 
1 Table 6. Sensitivity differences for different ages of NPC patients by using different markers

\begin{tabular}{|c|c|c|c|c|c|c|c|c|c|}
\hline \multirow{2}{*}{ Marker } & \multicolumn{8}{|c|}{ Sensitivity (\%) for different ages (years) of NPC patients } & \multirow{2}{*}{$\mathrm{P}^{\dagger}$} \\
\hline & $30 \sim$ & $35 \sim$ & $40 \sim$ & $45 \sim$ & $50 \sim$ & $55 \sim$ & $60 \sim$ & $65 \sim 69$ & \\
\hline \multicolumn{10}{|l|}{ VCA-IgA $\times$} \\
\hline & 100.00 & 85.71 & 89.29 & 91.30 & 85.11 & 100.00 & 93.55 & 90.48 & 0.274 \\
\hline \multicolumn{10}{|l|}{ EBNA1-IgA } \\
\hline PROB & 85.71 & 78.57 & 78.57 & 84.78 & 83.00 & 90.91 & 87.10 & 80.95 & 0.904 \\
\hline VCA-IgA & 78.57 & 78.57 & 85.71 & 80.43 & 78.72 & 84.85 & 87.10 & 76.19 & 0.950 \\
\hline EBNA1-IgA & 92.86 & 78.57 & 85.71 & 86.96 & 85.11 & 93.94 & 83.87 & 76.19 & 0.674 \\
\hline
\end{tabular}

2 The sensitivity differences in different ages of NPC patients were compared by Fisher's exact test. 


\section{Table 7 (on next page)}

Sensitivity differences for different smoking history NPC by using different markers 
1 Table 7. Sensitivity differences for different smoking history NPC by using different markers

\begin{tabular}{ccccc}
\hline \multirow{2}{*}{ Marker } & Cut-off value & \multicolumn{2}{c}{ Sensitivity (\%) for NPC } & \multirow{2}{*}{$\mathrm{P}^{\dagger}$} \\
\cline { 3 - 4 } & & smoking & no smoking & \\
\hline VCA-IgAx & 0.429 & 91.01 & 91.72 & 0.850 \\
EBNA1-IgA & & & & \\
PROB & 0.949 & 87.64 & 82.07 & 0.257 \\
VCA-IgA & 1.194 & 85.39 & 79.31 & 0.243 \\
EBNA1-IgA & 0.397 & 86.52 & 85.52 & 0.831 \\
\hline
\end{tabular}

2 The sensitivity differences for different smoking history NPC were compared by $\chi^{2}$ test. 


\section{Table 8 (on next page)}

Sensitivity differences for NPC with and without NPC family history by using different markers 
1 Table 8. Sensitivity differences for NPC with and without NPC family history by using different markers

\begin{tabular}{|c|c|c|c|c|}
\hline \multirow[b]{2}{*}{ Marker } & \multirow[b]{2}{*}{ Cut-off value } & \multicolumn{2}{|c|}{ Sensitivity (\%) } & \multirow[b]{2}{*}{$\mathrm{P}^{\dagger}$} \\
\hline & & $\begin{array}{l}\text { NPC with NPC } \\
\text { family history }\end{array}$ & $\begin{array}{l}\text { NPC without NPC } \\
\text { family history }\end{array}$ & \\
\hline \multicolumn{5}{|l|}{ VCA-IgAx } \\
\hline & 0.429 & 91.30 & 91.47 & 1.000 \\
\hline \multicolumn{5}{|l|}{ EBNA1-IgA } \\
\hline PROB & 0.949 & 78.26 & 84.83 & 0.412 \\
\hline VCA-IgA & 1.194 & 86.96 & 81.04 & 0.487 \\
\hline EBNA1-IgA & 0.397 & 73.91 & 87.02 & 0.082 \\
\hline
\end{tabular}

2 The sensitivity differences between NPC with and without NPC family history were compared by $\chi^{2}$ test. 
Figure 1

Comparison of levels of markers in NPC patients and healthy controls by t tests.

The dotted lines represent cut-off values of the markers. Each box indicates 25/75 percentiles. Whisker caps represent 10/90 percentiles. 

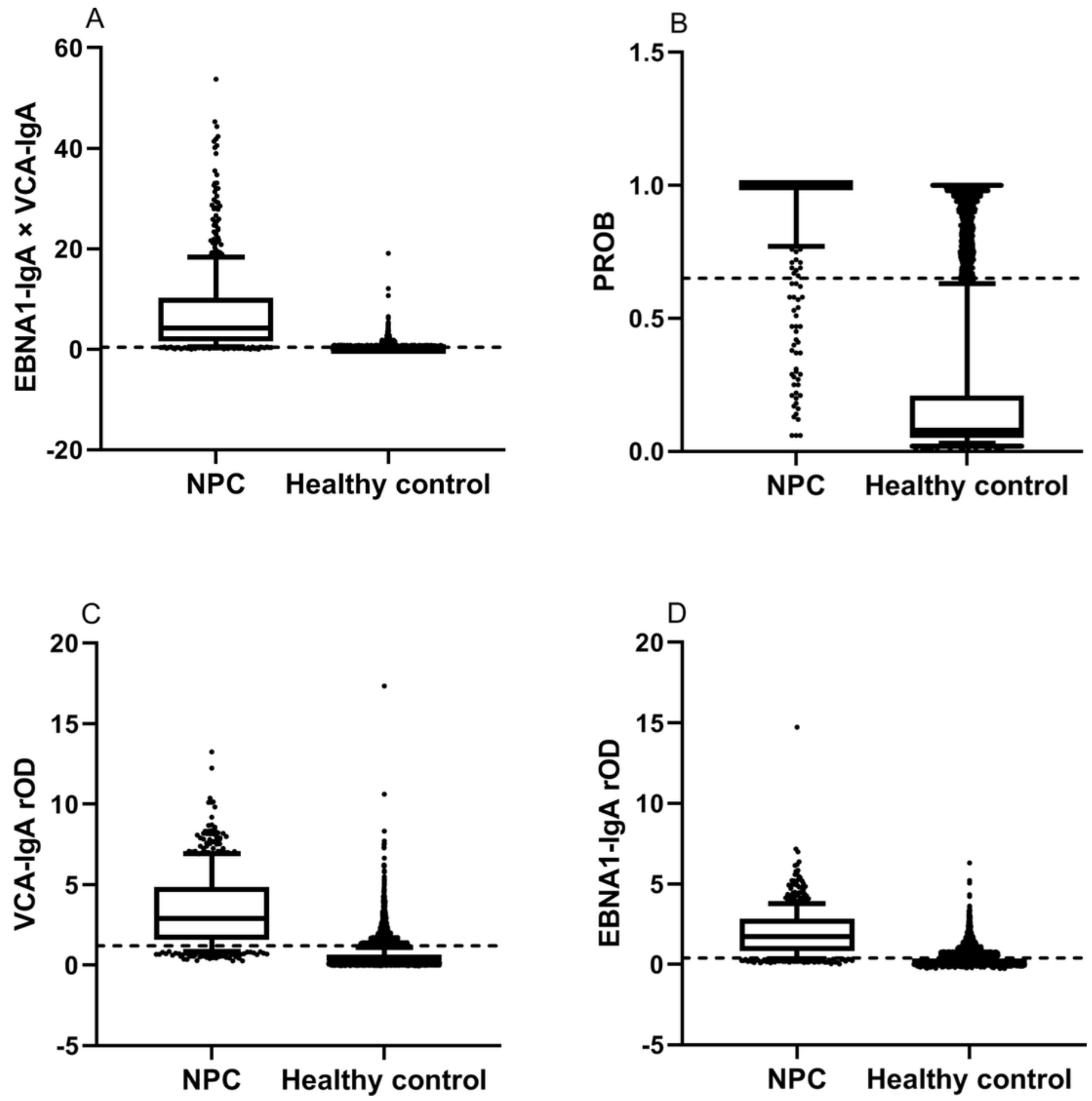
Figure 2

Comparison of levels of markers in early-stage and advanced-stage NPC patients by $\mathrm{t}$ tests.

The dotted lines represent cut-off values of the markers. Each box indicates 25/75 percentiles. Whisker caps represent 10/90 percentiles. 

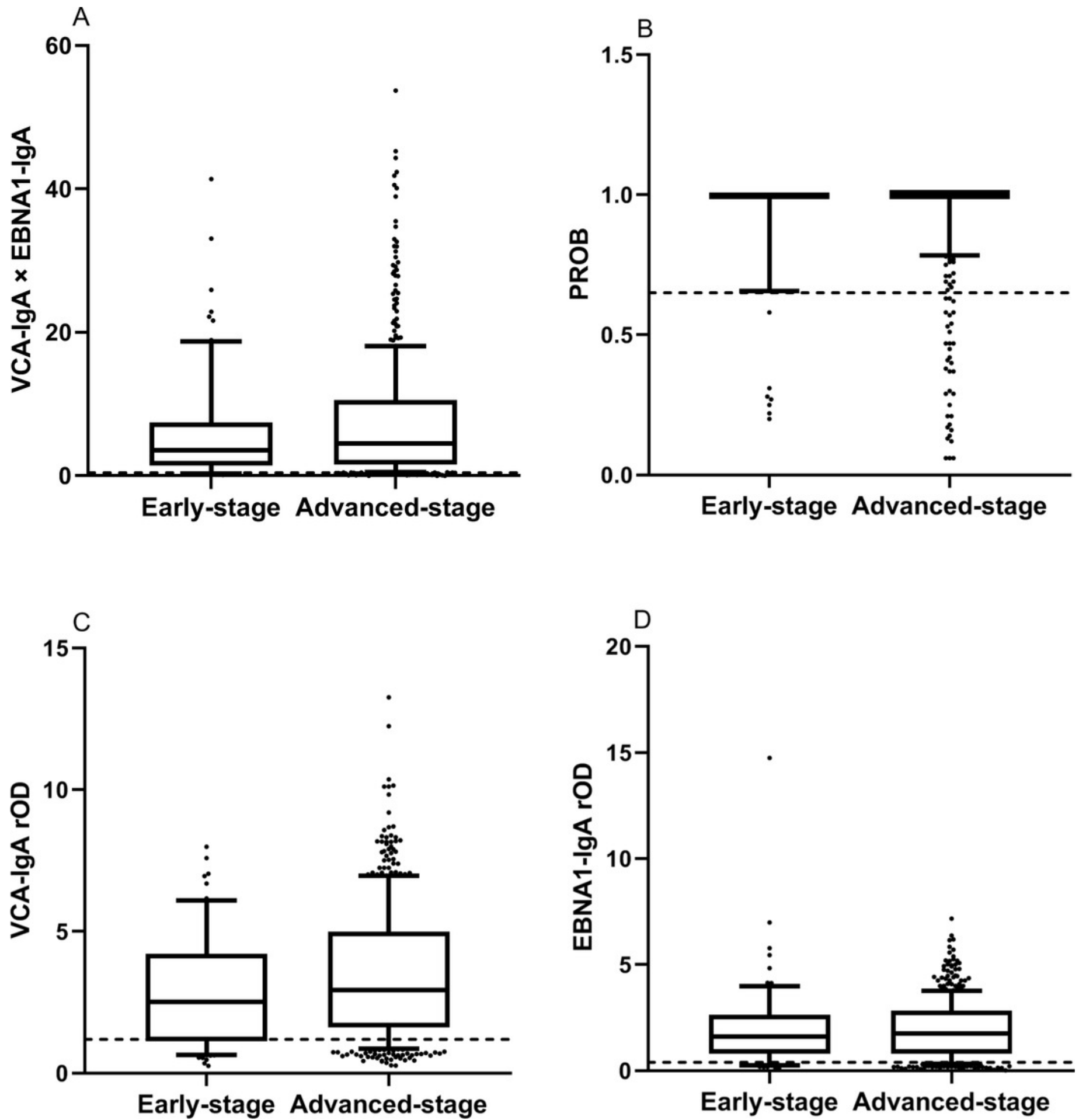
Figure 3

ROC curves for combination, PROB, VCA-IgA and EBNA1-IgA.

The axes are expressed as percentages.

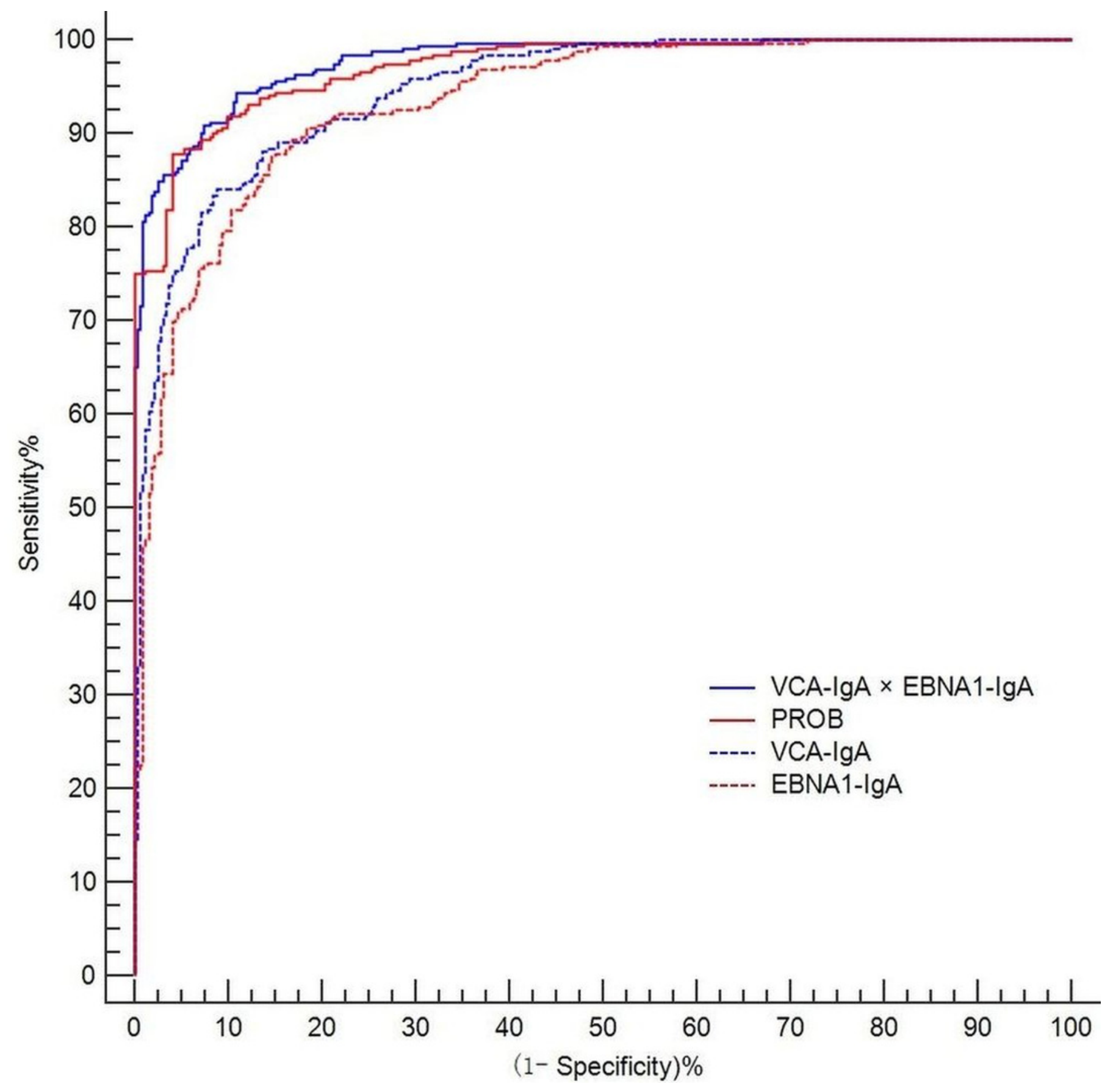

